\title{
Imperfect nesting and transport properties in unconventional density waves
}

\author{
Balázs Dóra \\ Department of Physics, Budapest University of Technology and Economics, H-1521 Budapest, Hungary \\ Kazumi Maki \\ Department of Physics and Astronomy, University of Southern California, Los Angeles CA 90089-0484, USA and \\ Max Planck Institute for the Physics of Complex Systems, \\ Nöthnitzer Str. 38, D-01187, Dresden, Germany \\ Attila Virosztek \\ Department of Physics, Budapest University of Technology and Economics, H-1521 Budapest, Hungary and \\ Research Institute for Solid State Physics and Optics, P.O.Box 49, H-1525 Budapest, Hungary
}

(Dated: November 6, 2018)

\begin{abstract}
We consider the effect of imperfect nesting in quasi-one dimensional unconventional density waves. The phase diagram is very close to those in a conventional DW. The linear and non-linear aspects of the electric conductivity are discussed. At $T=0$ the frequency dependent electric conductivity develops a small dip at low frequencies. The threshold electric field depends strongly on the imperfect nesting parameter, allowing us to describe the measured threshold electric field in the low temperature phase of the quasi-two dimensional organic conductor, $\alpha$-(BEDT-TTF $)_{2} \mathrm{KHg}(\mathrm{SCN})_{4}$ very well.

PACS numbers: 75.30.Fv, 71.45.Lr, 72.15.Eb, 72.15.Nj
\end{abstract}

\section{INTRODUCTION}

Unconventional density waves (i.e. density waves with momentum dependent gap) have long been suspected to be the possible ground state of certain materials. Since the original proposall, a number of studies have been done to discover the investigated的, and the stability of the different phases was studied 1 . In three dimensions, the phase diagram and the thermodynamic properties were discussed 3 . But certainly one of the main issues is the "rediscovery" of unconventional charge density waves with higher apoular momentum, the so-called d-density wave $\left(\Delta(\mathbf{k}) \sim \cos \left(k_{x} a\right)-\cos \left(k_{y} a\right)\right)$, which was investigated exhaustively f $_{\text {f }}$ over the past few years, and seems to convincingly describe many aspects of underdoped high $T_{c}$ superconductors currents around the plaquettes of the lattice, inducing some kind of magnetic order. This is why this phase was previously known as orbital antiferromagnet 9 .

Recently we have reported about different-spects of unconventional spin density waves (USDW) and unconventional charge densitivivaves (UCDW) 10.11 12.13 .14 in quasi-one dimensional systems. In analogy with unconventional superconductivity 5 . 16 , the extension of the conventional DW theory looks very natural to the case where the gap depends on the quasiparticle momentum like $\Delta(\mathbf{k}) \sim \sin \left(c k_{z}\right)$. In Ref. 10, a detailed description of the thermodynamics and optical conductivity of USDW is given. Interestingly, the UDW phase transition is not accompanied by a spatially periodic modulation of the spin or charge density. In Ref. 11, we studied the threshold electric field in perfectly nested UDW. In Ref. 12, 13, the effect of applied magnetic field is discussed, focusing on the phase diagram and the threshold electric field. In Ref. 14, the effect of impurities were treated in the Born limit.

On the other hand, the nature of the low temperature phase (LTP) of the $\alpha$-(ET) ${ }_{2} \mathrm{KHg}(\mathrm{SF} f)_{4}$ is not yet fully understood. In the LTP there is no X-ray or NMR signal-1,18 characteristic to conventional DWL1.20, which naturally follows our theory 10 . The destruction of the LTP in ipgreasing magnetic field suggests that it is a kind of a CDW 222122 , and the experimentally obtained phase diagram 19 is very close to our theoretical prediction in the Pauli limiting case in UCDW12.13.

Recently the threshold electric field associated with the sliding motion of DW of $\alpha$ - $(\mathrm{ET})_{2} \mathrm{KHg}(\mathrm{SCN})_{4}$ has been reported2324. It shows strong temperature dependence contray tho the one in conventional SDW, while it is not divergent at $T_{c}$ as one would expect from a conventional CDW2526. Our previous anafysis on the threshold electric field shows good agreement with the experimental data especially at ley temperatures1 12 . The strong, monotonic enhancement of the threshold electric field in an applied magnetic field 24 also coincides with our theory.

The Fermi surface of the $\alpha$-(ET) $)_{2}$ salts contains-large quasi-one dimensional sheets favouring the density wave phase transition, and small two dimensional pocket 227. Since the quasi-one dimensional sheets do not necessarily nest each other perfectly, it looks necessary to consider theoretically imperfectly nested systems to improve the matching between theory and experiment. As in conventional DW22,29, this effect can be handled in terms of imperfect nesting. 
In a recent paper, we succeeded in describing the measured angle dependent magnetoresistance data in $\alpha$-(ET) $)_{2}$ salt in the presence of imperfect nesting 30 . This is why we believe that the inclusion of imperfect nesting can help us to describe the threshold electric field as well.

The object of the present paper is to extend the earlier analysis 11 . 12 in the presence of imperfect nesting. We discuss the temperature dependence of the order parameter at different $\epsilon_{0}$ 's, where $\epsilon_{0}$ is the imperfect nesting parameter defined by the strongly anisotropic nearest neighbor tight-binding spectrum $\varepsilon(k)$ and by the nesting vector $\mathbf{Q}$ as $\varepsilon(\mathbf{k})+\varepsilon(\mathbf{k}-\mathbf{Q})=2 \epsilon_{0} \cos \left(2 b k_{y}\right)$. The phase boundary is almost the same as in a conventional DW. In the density of states the peaks at $\pm \Delta$ split into cusps at $\pm \Delta \pm \epsilon_{0}$. In the optical conductivity, only the low frequency part is modified due to imperfect nesting. The weight of the Dirac delta peak at zero frequency is finite at all temperatures. The threshold electric field depends strongly on $\epsilon_{0}$, and at $\epsilon_{0}=0.8 \Delta_{00} E_{T}$ shows very good agreement with the experimental data on $\alpha-(\mathrm{ET})_{2} \mathrm{KHg}(\mathrm{SCN})_{4} 23$. Therefore we may conclude that the LTP in $\alpha-(\mathrm{ET})_{2}$ salts is most likely an imperfectly nested UCDW. Some preliminary results have already been published in Ref. 31.

\section{PHASE DIAGRAM}

As a model we take the highly anisotropic quasi-one dimensional tight-binding Hamiltonian for the kinetic energy, and consider the interaction between the on-site and nearest neighbor electrons on orthogonal lattice 10 . The gap depends on the wavevector like $\Delta \sin \left(c k_{z}\right)$ or $\Delta \cos \left(c k_{z}\right)$. The effect of imperfect nesting is incorporated in the theory by replacing the Matsubara frequency with $\omega_{n}+i \epsilon_{0} \cos \left(2 b k_{y}\right) 28$,29. With this, the gap-equation reads as

$$
1=T \pi P \frac{N_{0}}{4} \sum_{n} \int_{0}^{2 \pi} \int_{0}^{2 \pi} \frac{\sin (z)^{2} d z d y}{\sqrt{\left(\omega_{n}+i \epsilon_{0} \cos (2 y)\right)^{2}+\Delta^{2} \sin (z)^{2}}},
$$

where $P$ is the interaction responsible for the UDW formation, $N_{0}$ is the density of states in the normal state at the Fermi energy per spin. From this, by taking $\Delta=0$, the second order phase boundary is given by

$$
1=T \pi P \frac{N_{0}}{4} \sum_{n} \frac{1}{\sqrt{\omega_{n}^{2}+\epsilon_{0}^{2}}} .
$$

This equation is identical to the BCS gap-equation for s-wave supereqnductor or conventional DW after replacing $\epsilon_{0}$ with $\Delta_{0}$, the order parameter of a perfect nested conventional DWE. From this, the second order phase boundary is given by $\epsilon_{0}=\Delta_{0}\left(T_{c}\right), \Delta_{0}(T)$ is the temperature dependence of the gap in a perfect nested conventional DW with $T_{c 0}$ transition temperature. The critical nesting is given by $\epsilon_{0}=\sqrt{e} \Delta_{00} / 2 \approx 0.82 \Delta_{00}$, where $\Delta_{00}$ is the gap in a perfectly nested system at zero temperature. At $T=0$ the order parameter remains unchanged for $2 \epsilon_{0}<\Delta_{00}$, then vanishes rapidly. This together with the phase diagram is shown in Fig. 11. The gap-equation was solved numerically for arbitrary temperatures, and the results are shown in Fig. 22.

\section{DENSITY OF STATES}

The density of states is given by

$$
N(E)=N_{0} \frac{4}{\pi^{2}} \int_{0}^{\pi / 2} \int_{0}^{\pi / 2} \operatorname{Re} \frac{\left|E-\epsilon_{0} \cos (2 y)\right|}{\sqrt{\left(E-\epsilon_{0} \cos (2 y)\right)^{2}-\Delta^{2} \sin ^{2}(z)}} d y d z=\frac{1}{\pi} \int_{0}^{\pi} \rho\left(E-\epsilon_{0} \cos y\right) d y,
$$

where $\rho(E)$ is the density of states in the perfectly nested system 10 and is the same as those in a d-wave superconductort5.

In Fig. 3, we show $N(E) / N_{0}$ for $\epsilon_{0} / \Delta=0,0.1,0.3,0.5$ and 0.8 . The inset shows the residual density of states, which is obtained as

$$
N(0)=N_{0} \frac{2}{\pi} \arcsin \frac{\epsilon_{0}}{\Delta}
$$

The peaks at $\pm \Delta$ split into cusps at $\pm \Delta \pm \epsilon_{0}$. As it is readily seen from Fig. 3 the DOS provides a clear signature of UDW with imperfect nesting. As a consequence of the finite number of occupied states at the Fermi energy, the specific heat increases linearly with temperature close to $T=0$ :

$$
C_{V}(T)=N(0) \frac{2 \pi^{2}}{3} k_{B}^{2} T
$$

which is in accordance with the measured specific heat in the $\alpha-(\mathrm{ET})_{2}$ salts32. 


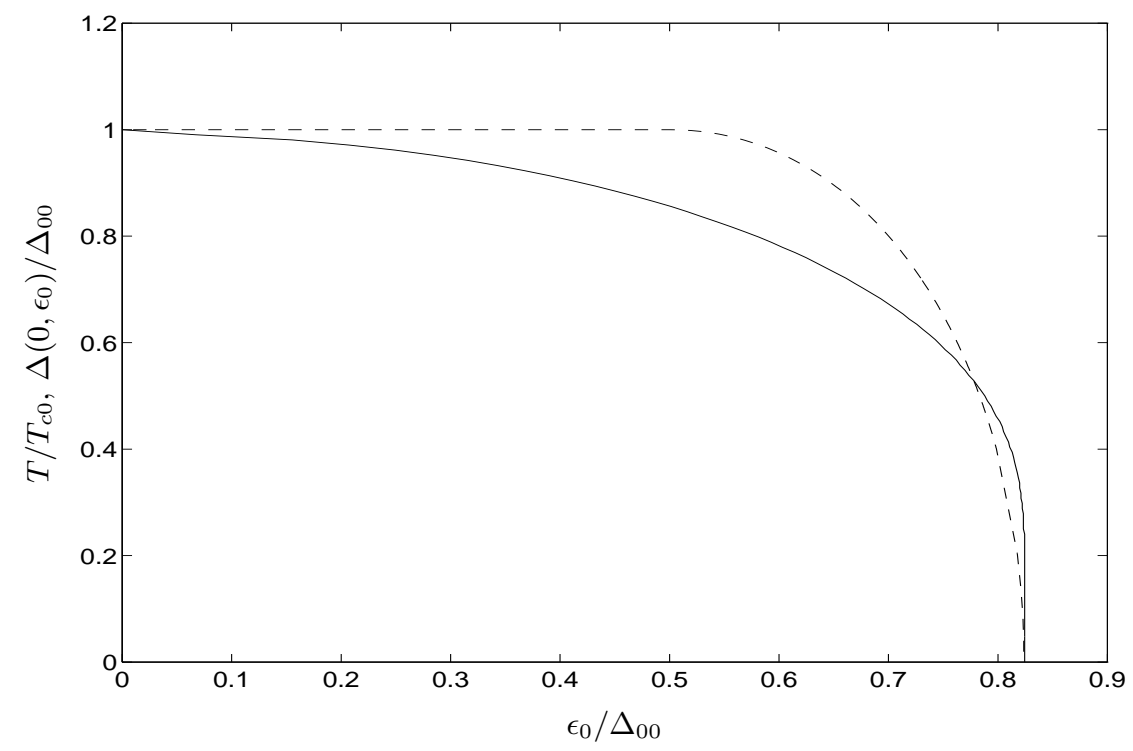

FIG. 1: The phase diagram (solid line) and the zero temperature order parameter (dashed line) are plotted in the presence of imperfect nesting.

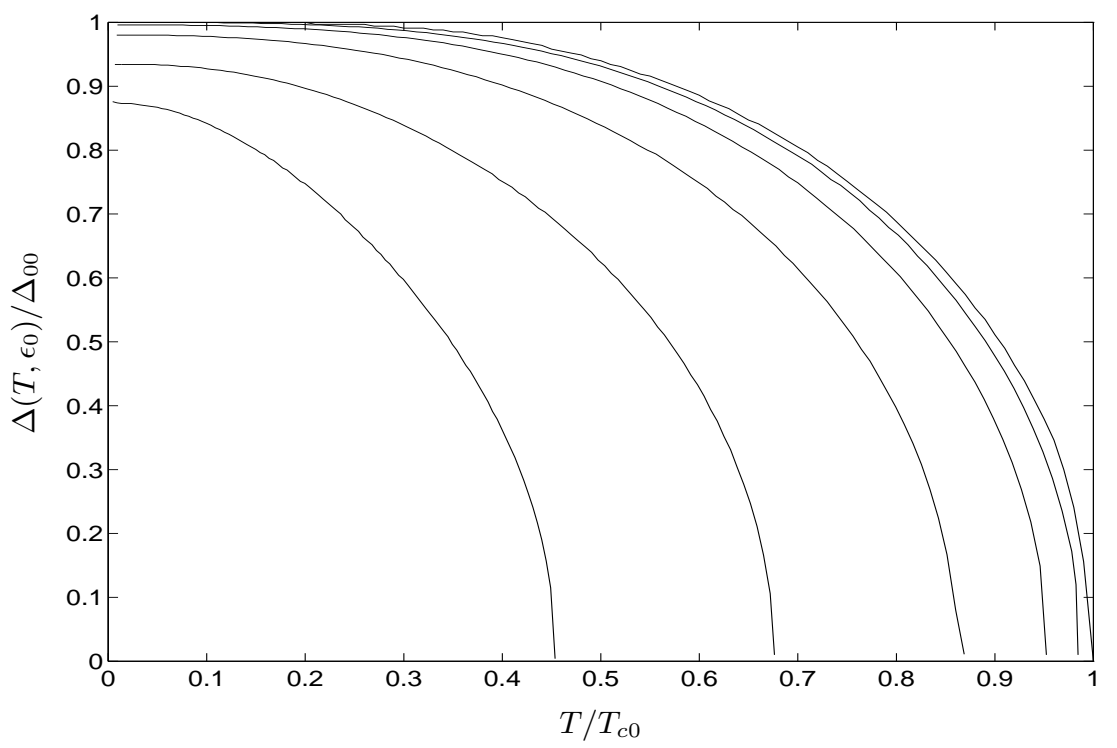

FIG. 2: The temperature dependence of the order parameter is shown for $\epsilon_{0} / \Delta_{00}=0,0.1,0.3,0.5,0.7$ and 0.8 from right to left.

\section{OPTICAL CONDUCTIVITY}

In this section we investigate the quasiparticle contribution to the optical conductivity. For simplicity we neglect the effect of the quasiparticle damping due to impurity scattering for example. The quasiparticle part of the conductivity contains relevant information about the system in the perpendicular cases ( $y$ and $z$ ) when the effect of the collective contributions can be neglected. The regular part of the optical conductivity is given by

$$
\operatorname{Re} \sigma_{\alpha \beta}^{r e g}(\omega)=N_{0} \frac{\pi e^{2}}{2 \omega^{2}} \int_{-\pi}^{\pi} \frac{d\left(b k_{y}\right)}{2 \pi} \int_{-\pi}^{\pi} \frac{d\left(c k_{z}\right)}{2 \pi} \operatorname{Re} \frac{v_{\alpha}(\mathbf{k}) v_{\beta}(\mathbf{k}) \Delta^{2}(\mathbf{k})}{\sqrt{(\omega / 2)^{2}-\Delta^{2}(\mathbf{k})}}\left(\tanh \left(\frac{|\omega|-2 \eta}{4 T}\right)+\tanh \left(\frac{|\omega|+2 \eta}{4 T}\right)\right),
$$




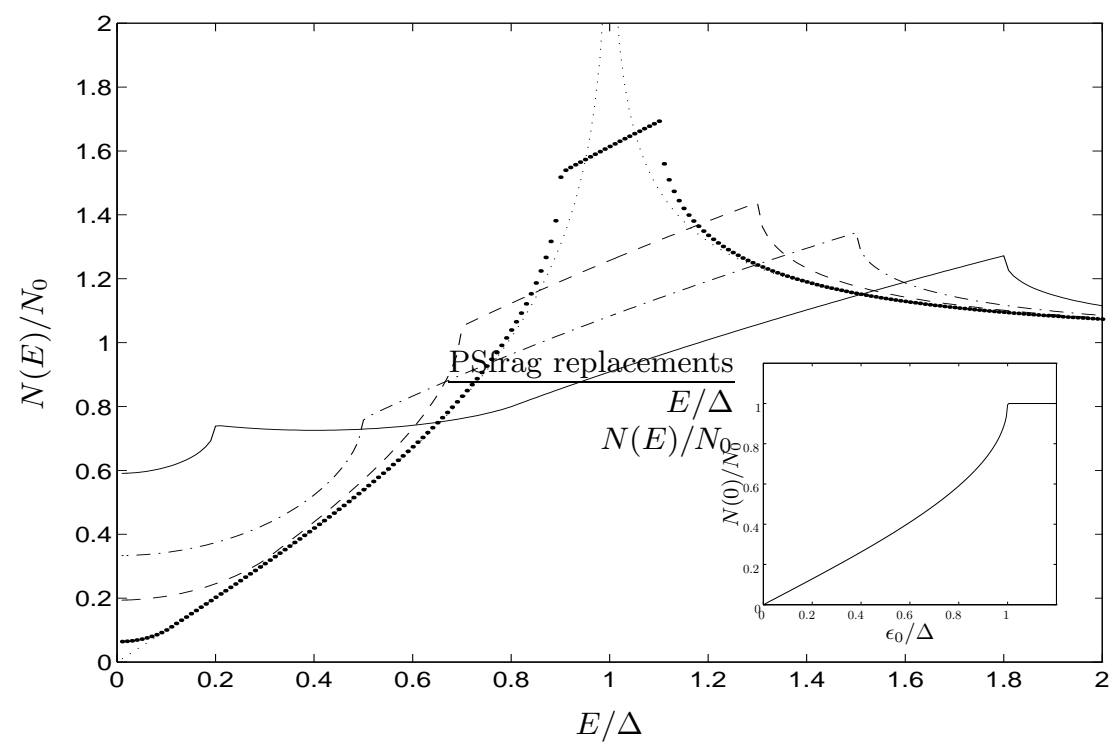

FIG. 3: The quasiparticle density of states is plotted as a function of energy for $\epsilon_{0} / \Delta=0$ (thin dotted line), 0.1 (thick dotted line), 0.3 (dashed line), 0.5 (dashed-dotted line) and 0.8 (solid line). The inset shows the residual density of states as a function of $\epsilon_{0} / \Delta$.

where $v_{\alpha}(\mathbf{k})$ is the quasiparticle velocity in the $\alpha$ direction, $\eta=\epsilon_{0} \cos \left(2 b k_{y}\right)$. From now on we restrict our investigation to the $T=0 \mathrm{~K}$ case. The optical conductivity remains unchanged for $\omega>2 \epsilon_{0}$ and is given by

$$
\operatorname{Re} \sigma\left(\omega, \epsilon_{0}\right)=\operatorname{Re} \sigma(\omega, 0) \frac{2}{\pi} \arcsin \left(\frac{\omega}{2 \epsilon_{0}}\right)
$$

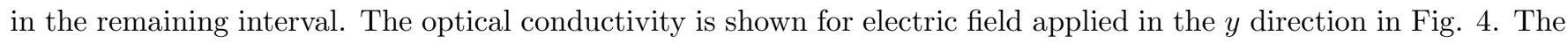
same curve belongs to both a sinusoidal or cosinusoidal gap function. In Figs. 5 and 6 , the optical conductivity is plotted in the $z$ direction for gap function $\Delta(\mathbf{k})=\Delta \sin \left(c k_{z}\right)$ and $\Delta(\mathbf{k})=\Delta \cos \left(c k_{z}\right)$, respectively. In this case, when the maximums of the velocity $\left(\sim \sin \left(c k_{z}\right)\right)$ and of the gap coincide, divergent peak shows up at $2 \Delta$. On the other hand, when the position of the gap maximum matches the zeros of the velocity, these excitations cannot produce any divergence. At first sight the sum rule seems to be violated since a lot of optical weight is missing at small frequencies. But the $\delta(\omega)$ part of the conductivity does not freeze out at $T \rightarrow 0$ in the presence of imperfect nesting, and its coefficient provides the missing area.

So far the optical conductivity of $\alpha-(\mathrm{ET})_{2} \mathrm{KHg}(\mathrm{SCN})_{4}$ salt has not been investigated comprehensively, only very few data are available at the moment 33 . For more decisive statements, more experiments are required especially in the low temperature regime.

\section{THRESHOLD ELECTRIC FIELD}

The behaviour of the $m_{\text {n }}$ linear conductivity is also of great interest. In terms of the phase $\Phi(\mathbf{r}, t)$ of DW the phase Hamiltonian is given by 25.26

$$
H(\Phi)=\int d^{3} r\left\{\frac{1}{4} N_{0} f\left[v_{F}^{2}\left(\frac{\partial \Phi}{\partial x}\right)^{2}+v_{b}^{2}\left(\frac{\partial \Phi}{\partial y}\right)^{2}+v_{c}^{2}\left(\frac{\partial \Phi}{\partial z}\right)^{2}+\left(\frac{\partial \Phi}{\partial t}\right)^{2}-4 v_{F} e E \Phi\right]+V_{i m p}(\Phi)\right\}
$$

where $f=\rho_{s}\left(T, \epsilon_{0}\right) / \rho_{s}(0,0), \rho_{s}\left(T, \epsilon_{0}\right)$ is the condensate density and $E$ is an electric field applied in the $x$ direction. Here $v_{F}, v_{b}$ and $v_{c}$ are the characteristic velocities of the quasi-one dimensional electron system in the three spatial directions. The condensate density is evaluated from the well-known formula:

$$
\rho_{s}=1-\frac{1}{4 T} \int_{-\infty}^{\infty} d E \frac{N(E)}{N_{0}} \operatorname{sech}^{2} \frac{E}{2 T} .
$$




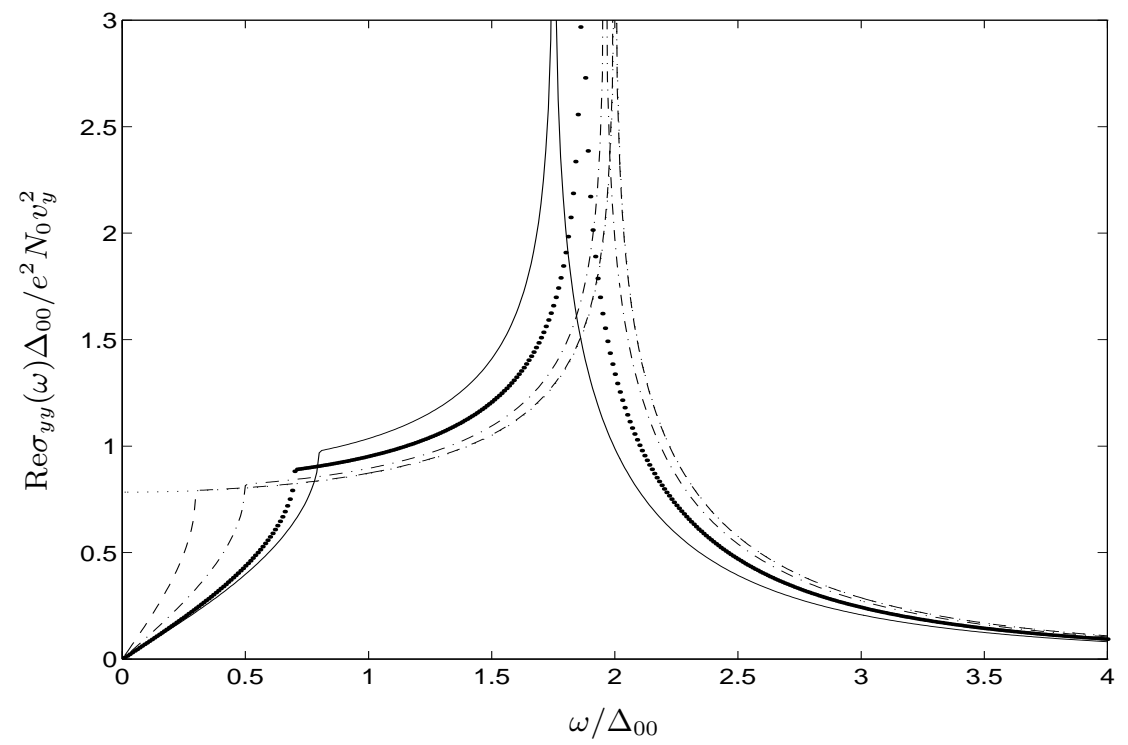

FIG. 4: The optical conductivity in the $y$ direction is shown for $\epsilon_{0} / \Delta\left(0, \epsilon_{0}\right)=0$ (thin dotted line), 0.3 (dashed line), 0.5 (dashed-dotted line), 0.7 (thick dotted line) and 0.8 (solid line).

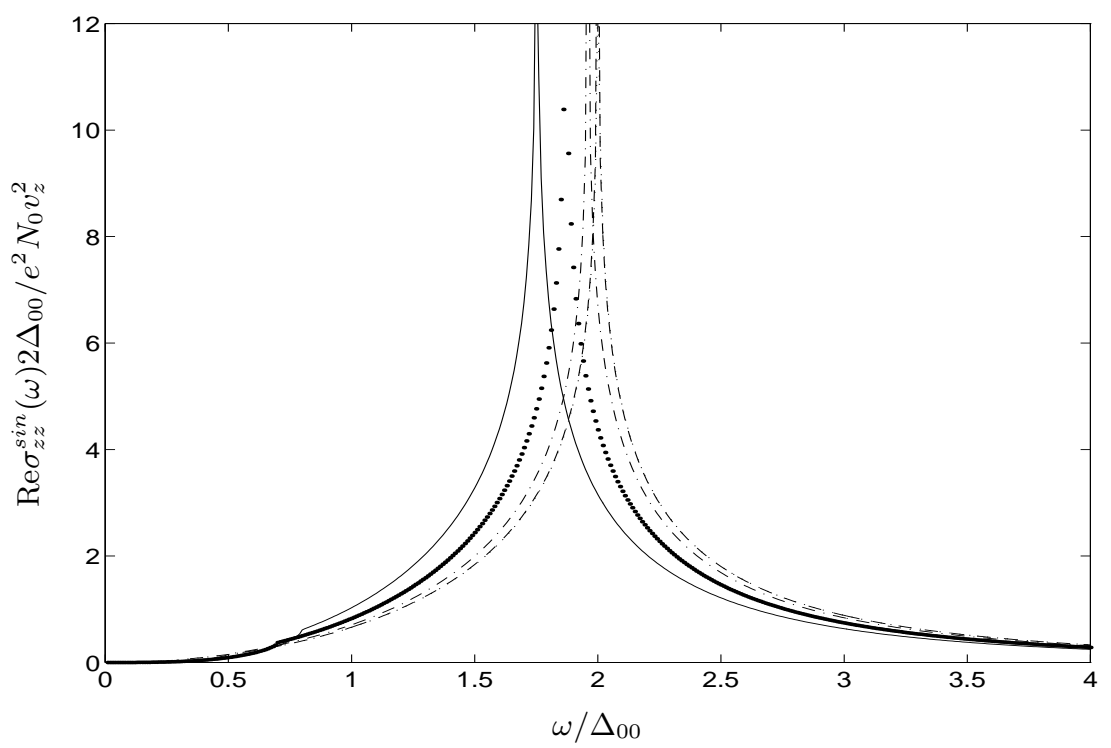

FIG. 5: The optical conductivity for a sinusoidal gap in the $z$ direction is shown for $\epsilon_{0} / \Delta\left(0, \epsilon_{0}\right)=0$ (thin dotted line), 0.3 (dashed line), 0.5 (dashed-dotted line), 0.7 (thick dotted line) and 0.8 (solid line).

At arbitrary temperatures it is evaluated numerically and shown in Fig. 7 for a set of $\epsilon_{0}$.

Assuming a nonlocal impurity potential as in Refs. 11,12

$$
U(\mathbf{Q}+\mathbf{q})=V_{0}+\sum_{i=y, z} V_{i} \cos \left(q_{i} \delta_{i}\right),
$$

the pinning potential is obtained as

$$
V_{i m p}(\Phi)=-\frac{8 V_{0} V_{y} N_{0}^{2}}{\pi} \sum_{j} \cos \left(2\left(\mathbf{Q} \mathbf{R}_{j}+\Phi\left(\mathbf{R}_{j}\right)\right)\right) \epsilon_{0} \int_{0}^{a+1} \tanh \frac{\epsilon_{0} x}{2 T} \frac{A B}{\pi^{2}} d x,
$$




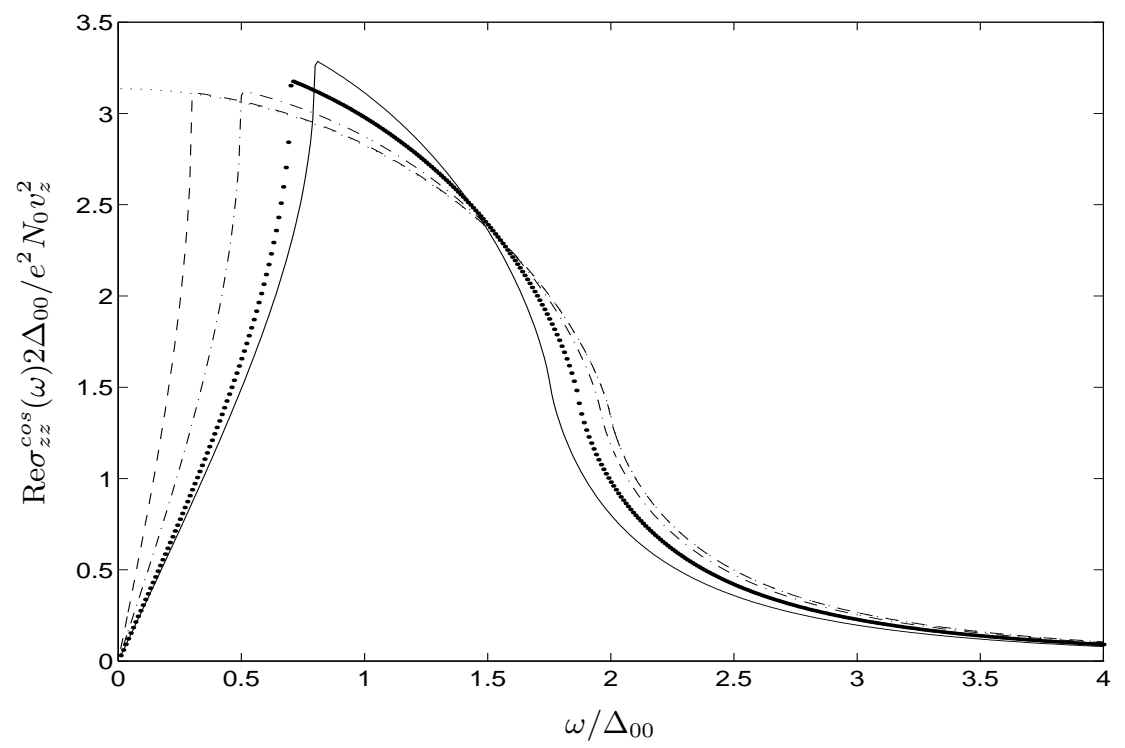

FIG. 6: The optical conductivity for a cosinusoidal gap in the $z$ direction is shown for $\epsilon_{0} / \Delta\left(0, \epsilon_{0}\right)=0$ (thin dotted line), 0.3 (dashed line), 0.5 (dashed-dotted line), 0.7 (thick dotted line) and 0.8 (solid line).

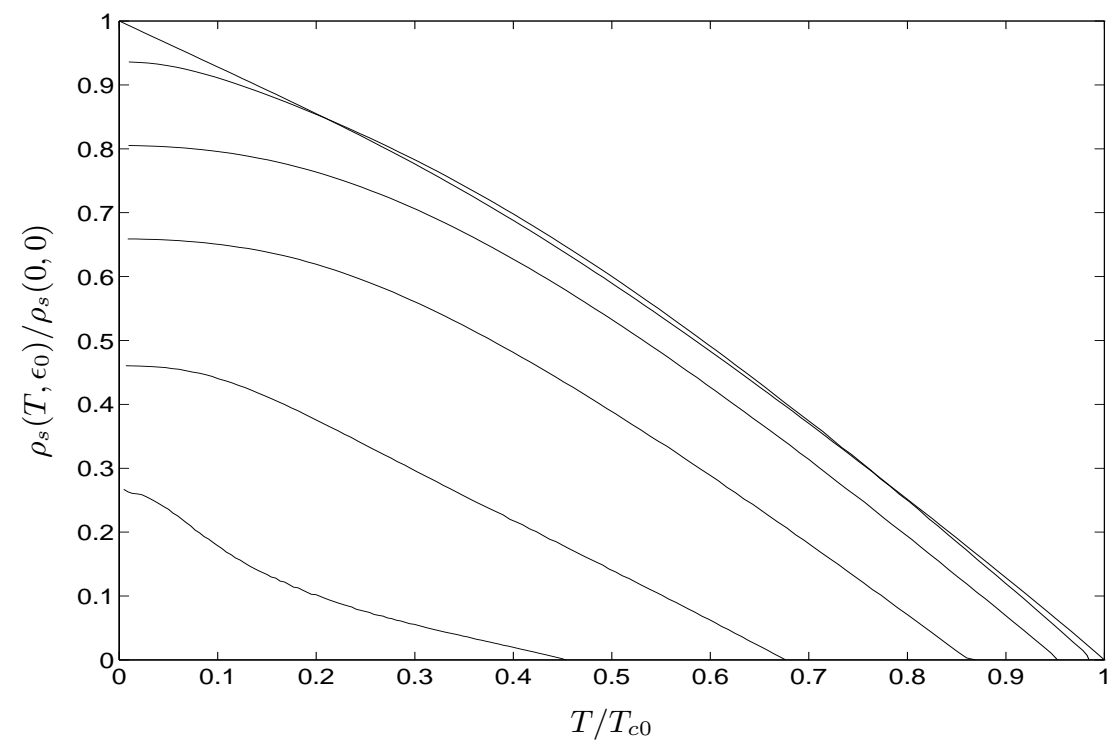

FIG. 7: The condensate density is shown as a function of temperature for $\epsilon_{0} / \Delta_{00}=0,0.1,0.3,0.5,0.7$ and 0.8 from top to bottom. 
where

$$
\begin{gathered}
A=\int_{\gamma}^{\delta} E\left(\sqrt{1-\left(\frac{x-\cos (y)}{a}\right)^{2}}\right) d y, \\
B=\int_{\gamma}^{\delta} \operatorname{sgn}(x-\cos (y))\left[K\left(\frac{x-\cos (y)}{a}\right)-E\left(\frac{x-\cos (y)}{a}\right)\right] d y+ \\
+\Theta(\pi-\delta) \int_{\delta}^{\pi} \frac{|x-\cos (y)|}{a}\left[K\left(\frac{a}{x-\cos (y)}\right)-E\left(\frac{a}{x-\cos (y)}\right)\right] d y+ \\
+\Theta(\gamma) \int_{0}^{\gamma} \frac{|x-\cos (y)|}{a}\left[K\left(\frac{a}{x-\cos (y)}\right)-E\left(\frac{a}{x-\cos (y)}\right)\right] d y \\
\gamma=\arccos \left(1-2 \max \left(0, \frac{1-a-x}{2}\right)\right), \\
\delta=\arccos \left(1-2 \min \left(1, \frac{a+1-x}{2}\right)\right)
\end{gathered}
$$

$a=\Delta / \epsilon_{0}$. Then following FLR 3435 , the threshold electric field in the strong pinning limit reads as

$$
\frac{E_{T}^{S}\left(T, \epsilon_{0}\right)}{E_{T}^{S}(0,0)}=\frac{\rho_{s}(0,0)}{\rho_{s}\left(T, \epsilon_{0}\right)} \frac{\epsilon_{0}}{0.5925} \int_{0}^{a+1} \tanh \frac{\epsilon_{0} x}{2 T} \frac{A B}{\pi^{2}} d x
$$

For any finite $\epsilon_{0}$ the density of states has no zero at the Fermi energy (Eq. (4)), and the threshold electric field increases as

$$
\frac{E_{T}^{S}(T, h)}{E_{T}^{S}(0,0)}= \begin{cases}1+2 \ln (2) \frac{T}{\Delta_{00}} & \epsilon_{0} \ll T \\ 1+\frac{2 \epsilon_{0}}{\pi \Delta_{00}} & T \ll \epsilon_{0}\end{cases}
$$

in the $\epsilon_{0}, T \ll \Delta_{00}$ range. The threshold electric field along the second order phase boundary is obtained as

$$
\frac{E_{T}^{S}\left(T, \epsilon_{0}\right)}{E_{T}^{S}(0,0)}=-\frac{\tanh \frac{\epsilon_{0}}{2 T}}{\left\langle\Psi^{\prime \prime}\left(\frac{1}{2}+\frac{i \epsilon_{0} \cos y}{2 \pi T}\right)\right\rangle} \frac{T^{2}}{\Delta_{00} \epsilon_{0}} \frac{\pi^{5}}{4 \times 0.5925} .
$$

The threshold electric field is shown in Fig. 8. Close to the critical nesting where the transition temperature tends to zero, threshold electric field diverges at the actual transition temperature as

$$
\frac{E_{T}^{S}\left(T, \epsilon_{0}\right)}{E_{T}^{S}(0,0)}=4.18 \frac{T^{2}}{\Delta_{00} \epsilon_{0}} \exp \left(1.03 \frac{\epsilon_{0}}{T}\right) .
$$

The strong pinning limit applies when impurities are introduced by X-ray irradiation or some violent means. In this case one single impurity is enough to pin the DW locally. For high quality crystals however, the weak pinning limit is more appropriate 36 . Then for the 3 dimensional weak pinning limit we obtain 25,26

$$
\frac{E_{T}^{W}\left(T, \epsilon_{0}\right)}{E_{T}^{W}(0,0)}=\left(\frac{E_{T}^{S}\left(T, \epsilon_{0}\right)}{E_{T}^{S}(0,0)}\right)^{4}
$$

$E_{T}^{W}\left(T, \epsilon_{0}\right)$ is plotted for $\epsilon_{0}=0.8 \Delta_{00}$ as a function of temperature together with the experimentaldata on $\alpha$ $(\mathrm{ET})_{2} \mathrm{KHg}(\mathrm{SCN})_{4} 23$ in Fig. 9. The fit was made with the least square method. As we have foreseen 11 , imperfect nesting has improved the agreement remarkably well. The present theoretical results apply also for USDW.

The agreement between the experimental and theoretical threshold electric field data, together with the magnetic field-temperature phase diagram12 19, the specific heat and the lack of any spatially periodic density modulation suggests, that the LTP of the $\alpha$-(BEDT-TTF $)_{2} \mathrm{KHg}(\mathrm{SCN})_{4}$ salt is probably UCDW. 


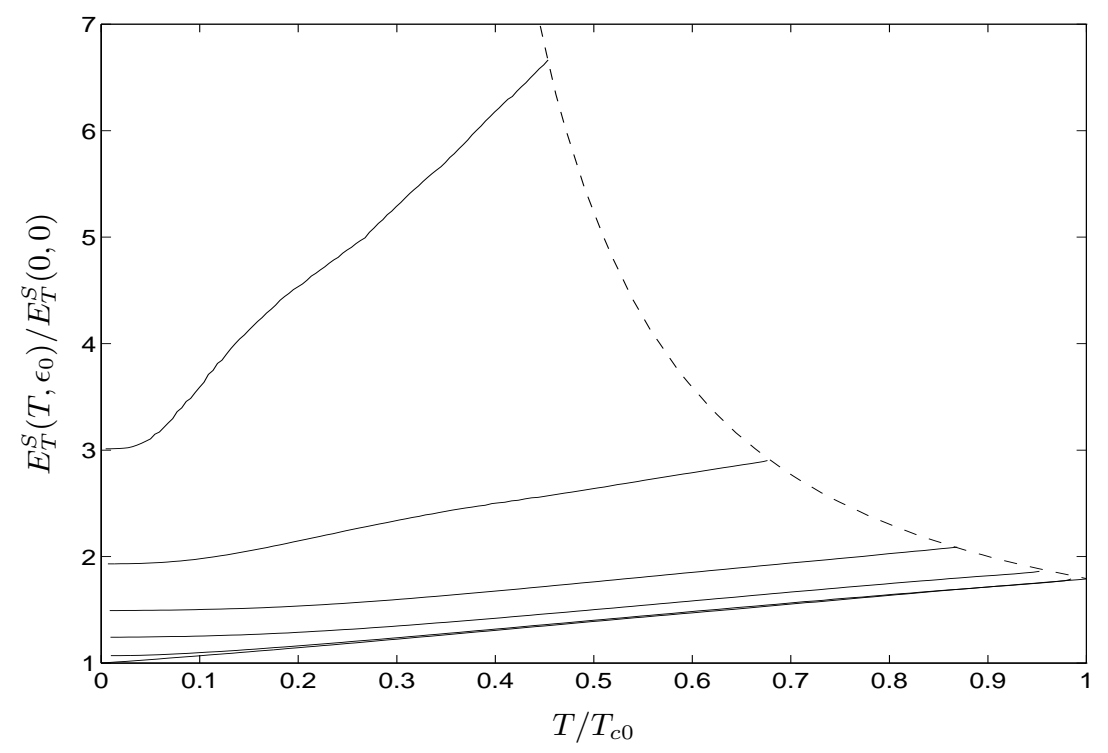

FIG. 8: The threshold electric field in the strong pinning limit is plotted for as a function of temperature for $\epsilon_{0} / \Delta_{00}=0,0.1$, 0.3, 0.5, 0.7 and 0.8 from bottom to top. The dashed line represents $E_{T}$ along the phase boundary.

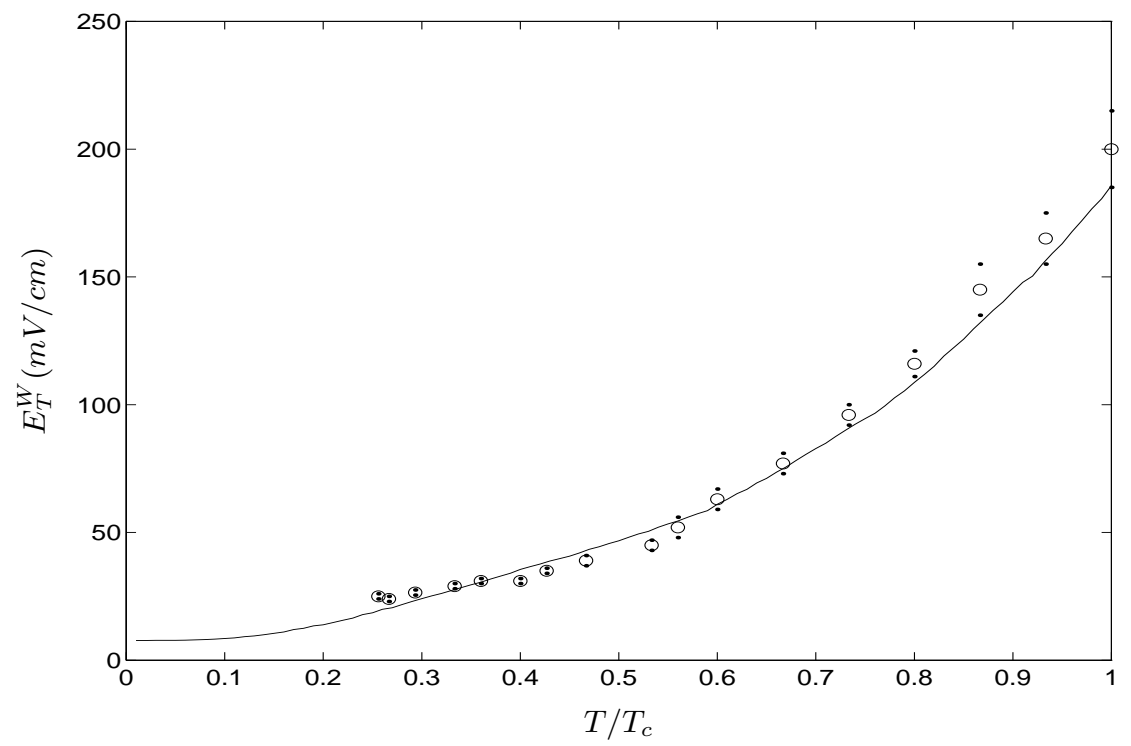

FIG. 9: The threshold electric field in the weak pinning limit is plotted as a function of reduced temperature for $\epsilon_{0}=0.8 \Delta_{00}=0$ together with the experimental data (open circles). The dots represent the error bar.

\section{CONCLUSION}

We have studied theoretically the effect of imperfect nesting in unconventional density waves. We explored the phase diagram which is identical to the one in conventional density wave. In the density of states, the peaks at $\pm \Delta$ of the perfectly nested system split into cusps at $\pm \Delta \pm \epsilon_{0}$. Usually $\epsilon_{0}$ is thought to vary with pressure providing the opportunity to check these result in a wide range of parameters. For example, the density of states obtained from ARPES measurements at different applied pressures can be compared to our predictions. Only the low frequency part of the conductivity is chopped transferring spectral weight to the Dirac delta peak at $\omega=0$ (similarly to the effect of magnetic field 2 ), which is expected to be broadened due to impurity effects. The threshold field shows similar 
features to the gne in conventional DW, increases smoothly with $\epsilon_{0}$ allowing us to describe the the measured $E_{T}$ of $\alpha-(\mathrm{ET})_{2}$ salts 23 with $\epsilon_{0}=0.8 \Delta_{00}$. This supports our proposal that the LTP of $\alpha-(\mathrm{ET})_{2}$ salts is an unconventional charge density wave.

\section{Acknowledgments}

We thank Bojana Korin-Hamzić for sending us the experimental data before publication which provided us with initial stimulus to undertake this work. We thank also Mark Kartsovnik, Takahiko Sasaki and Peter Thalmeier for useful discussions. This work was supported by the Hungarian National Research Fund under grant numbers OTKA T032162 and T037451, and by the Ministry of Education under grant number FKFP 0029/1999.

1 B. I. Halperin and T. M. Rice, in Solid State Physics, edited by F. Seitz, D. Turnbull, and H. Ehrenreich (Academic Press, New York, 1968), vol. 21, p. 115.

2 A. A. Nersesyan and G. E. Vachnadze, J. Low T. Phys. 77, 293 (1989).

3 A. A. Nersesyan, G. I. Japaridze, and I. G. Kimeridze, J. Phys. Cond. Mat. 3, 3353 (1991).

${ }^{4}$ H. J. Schulz, Phys. Rev. B 39, 2940 (1989).

5 Zs. Gulácsi and M. Gulácsi, Phys. Rev. B 36, 699 (1987).

6 S. Chakravarty, R. B. Laughlin, D. K. Morr, and C. Nayak, Phys. Rev. B 63, 094503 (2001).

7 W. Kim and J. P. Carbotte, cond-mat/0202514.

8 L. Benfatto, S. Caprara, and C. Di Castro, Eur. Phys. J. B 17, 95 (2000).

${ }^{9}$ M. Ozaki, Int. J. Quantum Chem. 42, 55 (1992).

10 B. Dóra and A. Virosztek, Eur. Phys. J. B 22, 167 (2001).

11 B. Dóra, A. Virosztek, and K. Maki, Phys. Rev. B 64, 041101(R) (2001).

12 B. Dóra, A. Virosztek, and K. Maki, Phys. Rev. B 65, 155119 (2002).

13 B. Dóra, A. Virosztek, and K. Maki, Curr. Appl. Phys. 1, 313 (2001).

14 B. Dóra, A. Virosztek, and K. Maki, cond-mat/0203434, Phys. Rev. B (in press).

15 H. Won and K. Maki, in Symmmetry and Pairing in Superconductors, edited by M. Ausloos and S. Kruchinin (Kluwer, Dordrecht, 1999).

16 C. C. Tsuei and J. R. Kirtley, Rev. Mod. Phys. 72, 969 (2000).

17 F. L. Pratt, T. Sasaki, N. Toyota, and K. Nagamine, Phys. Rev. Lett. 74, 3892 (1995).

18 K. Miyagawa, A. Kawamoto, and K. Kanoda, Phys. Rev. B 56, R8487 (1997).

19 P. Christ, W. Biberacher, M. V. Kartsovnik, E. Steep, E. Balthes, H. Weiss, and H. Müller, JETP Lett. 71, 303 (2000).

20 J. Singleton, Rep. Prog. Phys. 63, 1161 (2000).

21 D. Zanchi, A. Bjelis, and G. Montambaux, Phys. Rev. B 53, 1240 (1996).

22 A. Bjelis, D. Zanchi, and G. Montambaux, J. Phys. IV France 9, Pr10 203 (1999).

23 M. Basletić, B. Korin-Hamzić, M. V. Kartsovnik, and H. Müller, Synth. Met. 120, 1021 (2001).

24 T. Fujita, T. Sasaki, N. Yoneyama, N. Kobayashi, and T. Fukase, Synth. Met. 120, 1077 (2001).

25 K. Maki and A. Virosztek, Phys. Rev. B 39, 9640 (1989).

${ }^{26}$ K. Maki and A. Virosztek, Phys. Rev. B 42, 655 (1990).

27 T. Sasaki and N. Toyota, Phys. Rev. B 49, 10120 (1994).

28 X. Huang and K. Maki, Phys. Rev. B 42, 6498 (1990).

29 X. Huang and K. Maki, Phys. Rev. B 46, 162 (1992).

30 B. Dóra, K. Maki, B. Korin-Hamzić, M. Basletic, A. Virosztek, M. V. Kartsovnik, and H. Müller, cond-mat/0204321, submitted to Europhys. Lett.

31 B. Dóra, A. Virosztek, and K. Maki, Physica B 312-313, 571 (2002).

32 Y. Nakazawa, A. Kawamoto, and K. Kanoda, Phys. Rev. B 52, 12890 (1995).

33 M. Dressel, N. Drichko, J. Schlueter, and J. Merino, cond-mat/0206074.

34 H. Fukuyama and P. A. Lee, Phys. Rev. B 17, 535 (1978).

35 P. A. Lee and T. M. Rice, Phys. Rev. B 19, 3970 (1979).

36 see for example S. Tomić, J. R. Cooper, W. Kang, D. Jerome, and K. Maki, J. Phys. I (Paris) 1, 1603 (1991). 